\title{
Experimental discrimination of plowing friction and shear friction
}

\author{
J.-D. Kamminga a and G.C.A.M. Janssen ${ }^{\mathrm{b}, *}$ \\ a 39 Quai Lucien Lefranc, 93303, Aubervilliers Cedex, France \\ ${ }^{b}$ Department of Materials Science and Engineering, Delft University of Technology, Mekelweg 2, 2628 CD, Delft, The Netherlands
}

Received 14 August 2005; accepted 6 April 2006; published online 19 December 2006

\begin{abstract}
The friction of a diamond spherical indenter sliding on $\mathrm{CrN}$ coated nitrided steel was investigated. A friction model was proposed that takes into account plowing and shear friction. With the model the separate contributions of substrate properties and surface condition to the friction were successfully extracted: the shear friction coefficient $\mu_{\mathrm{sh}}$ was found to depend exclusively on the surface condition, i.e., not on load on the indenter, hardness of the substrate, and thickness of the coating. On the other hand, the plowing component of friction was independent on surface condition.
\end{abstract}

KEY WORDS: sliding friction, plowing, elastic recovery, scratch test, $\mathrm{CrN}$ coating

The friction experienced by a hard indenter plowing through a relatively soft material consists of two contributions. The plastic deformation of the soft specimen yields a plowing friction component while the shear forces at the area of contact yield shear friction [1-3]. For coated specimens with a relatively thin coating as compared to the penetration depth of the indenter, the plowing term is governed by the plastic properties of the substrate, and coating properties control shear friction. In several publications the combined plowing and shear friction of a hard indenter plowing through a softer counterpart was discussed $[1,4,5]$. In these publications, the material under investigation was assumed to be perfectly plastic. Thereby, elastic recovery of the material trailing the sliding indenter was neglected and, as a consequence, the normal load was assumed to be carried exclusively by the front half of the indenter. However, in recent articles elastic recovery was shown to play an important role in the friction of sliding indenters [6-8]. We propose an analysis of friction, which takes into account elastic recovery.

Sliding friction of an indenter with a diamond spherical tip on nitrided steel covered with a $\mathrm{CrN}$ coating was investigated. The combined nitriding-coating surface treatment is intended to improve the tribological performance of the steel $[9,10]$. The $\mathrm{CrN}$ coating provides low friction and good abrasive wear resistance, while the steel (hardened by nitriding) yields high load carrying capacity and fatigue resistance. With a detailed analysis of the friction of the indenter sliding on such specimens we intend to assess the separate contributions

*To whom correspondence should be addressed.

E-mail: G.C.A.M.Janssen@tudelft.nl of coating and nitrided substrate to the overall mechanical properties. This is crucial for optimization of coated-nitrided steels in practical applications.

A spherical indenter sliding on a flat surface is considered (sliding direction $x$, normal load applied along the $z$-direction). The normal load $F_{\mathrm{N}}$ connects the scratch hardness $H_{\mathrm{sc}}$ and the geometrical contact area $A_{z}$ projected in the $z$-direction through $F_{\mathrm{N}}=H_{\mathrm{so}} A_{z}$ [10,11]. Similarly, it holds $F_{\mathrm{pl}}=H_{\mathrm{pl}} A_{x}$ (plowing force $F_{\mathrm{pl}}$, plowing hardness $H_{\mathrm{pl}}$, geometrical contact area projected in the $x$-direction $A_{\mathrm{x}}$ ). Both $H_{\mathrm{sc}}$ and $H_{\mathrm{pl}}$ are associated with the resistance of the specimen against plastic deformation and are approximately equal [4]. The shear friction force $F_{\text {hs }}$ assumed to obey Amontons friction law: $F_{\mathrm{sh}}=\mu_{\mathrm{sh}} F_{\mathrm{N}}$ (shear friction coefficient $\mu_{\mathrm{sh}}$ ) $[2,3]$. Assuming further that the total friction force $F_{\mathrm{f}}$ equals the sum of $F_{\mathrm{sh}}$ and $F_{\mathrm{pl}}$, it follows:

$$
\frac{F_{\mathrm{f}}}{F_{\mathrm{N}}}=\mu_{\mathrm{sh}}+\frac{H_{\mathrm{pl}}}{H_{\mathrm{sc}}} \frac{A_{x}}{A_{z}} \approx \mu_{\mathrm{sh}}+\frac{A_{x}}{A_{z}}
$$

As will be shown, an approximately Hertzian contact exists between the indenter and the specimen. Consequently, the area of contact projected in the $z$-direction is approximately circular, radius $a, A_{z}=\pi a^{2}$ (because the stress is concentrated in the center of the contact [12-14], small deviations from a circular projected contact area possibly present in the trailing half of the indenter do not considerably influence the analysis of friction). Since plowing is associated with plastic deformation, values for $A_{x}$ and $A_{z}$ after unloading are used for the analysis of friction (such, if the deformation is purely elastic, $A_{x}$, after unloading is zero and $\left.F_{\mathrm{f}} / F_{\mathrm{N}}=\mu_{\mathrm{sh}}\right)$. 
$A_{z}$ remains approximately constant upon unloading [12]. However, if the material is not perfectly plastic, $A_{x}$ decreases considerably during unloading. In order to estimate the value of $A_{x}$ after unloading (i.e., the residual cross-section of the track left after unloading) from the track width, a Hertzian displacement field will be used. For a static spherical indenter, the permanent indentation left in the specimen surface after unloading has a radius of curvature $R_{\text {unl }}$ larger than that of the indenting sphere $R_{\text {ind }}$ [12]. If unloading occurs elastically, $R_{\text {unl }}$ is connected to $a, R_{\text {ind }}, F_{\mathrm{N}}$, and the elastic constants (Young's modulus E, Poisson's ratio $v$ ) through $[12,13]$ :

$$
a^{3}=\frac{3 F_{\mathrm{N}} R_{\text {ind }} R_{\text {unl }}}{4\left(R_{\text {unl }}-R_{\text {ind }}\right)}\left(\frac{1-v_{\text {ind }}^{2}}{E_{\text {ind }}}+\frac{1-v^{2}}{E}\right)
$$

In this work, equation (2) will be shown to approximately hold also for a sliding indenter. For the analysis of the friction of the sliding indenter on the basis of equation (1), $A_{x} / A_{z}$ is expressed in terms of $a$ and $R_{\mathrm{unl}}$, using straightforward geometry $\left(A_{z}=\pi a^{2}, A_{x}\right.$ is the cross-sectional area for a groove with radius of curvature $R_{\mathrm{unl}}$ and width $2 a$ ):

$$
\frac{A_{x}}{A_{z}}=\frac{1}{\pi}\left(\frac{R_{\mathrm{unl}}}{a}\right)^{2} \arcsin \left(\frac{a}{R_{\mathrm{unl}}}\right)-\frac{1}{\pi} \frac{R_{\mathrm{unl}}}{a} \sqrt{1-\left(\frac{a}{R_{\mathrm{unl}}}\right)^{2}}
$$

For perfectly plastic material $R_{\text {ind }}$ equals $R_{\text {unl }}$ Then equation (3) holds with $R_{\text {unzl }}=R_{\text {ind }}$ and projected contact area $A_{z, \text { plast }}=A_{z} / 2$ (see Refs. [1,4,5]).

Steel nitriding and $\mathrm{CrN}$ coating deposition were performed in a HTC 750 PVD apparatus (for a detailed description of the nitriding and deposition procedures see Ref. [9]). A set of steel substrates was nitrided employing various nitriding times (15-480 min). Upon increasing the nitriding time the scratch hardness increased from 5 to $8 \mathrm{GPa}$ [10]. On one of the nitrided steels (nitriding time $1 \mathrm{~h}$ ), also a $100 \mathrm{~nm} \mathrm{CrN}$ coating was deposited. Additionally, one of the specimens was covered with a solid lubricant (a suspension was sprayed on the specimen that was subsequently dried) [15]. It was verified that the solid lubricant layer was thin enough not to affect the plowing friction component (see below). A diamond indenter with a spherical tip (radius $200 \mu \mathrm{m}$ ) was slid over the specimens using a CSM Revetest scratch tester. Sliding experiments were performed at a constant normal load (range $10-80 \mathrm{~N}$, sliding speed $5 \mathrm{~mm} / \mathrm{min}$, track length $5 \mathrm{~mm}$ ). The friction force was measured. An optical microscope was used to measure the track width and to verify that the $\mathrm{CrN}$ coating was not removed from the specimens. The track widths were in the range from 40 to $160 \mu \mathrm{m}$. Scratch track depth profiles (cross sections) were obtained with a confocal laser-scanning microscope.
From the depth profiles the projected area $A_{x}$ was obtained by numerical integration.

The upper curve in figure 1 shows a cross section of a scratch track in a $\mathrm{CrN}$ coated nitrided specimen (thickness $1 \mu \mathrm{m}$, nitriding time $1 \mathrm{~h}$, load $60 \mathrm{~N}$ ). The lower curve (solid line) shows the track depth profile obtained by adding to the unloaded track depth profile the Hertzian displacement field of a static spherical indenter [14] (load and indenter diameter equal to the experimental values). In the calculation the indenter was taken infinitely stiff (Young's modulus of diamond: $1100 \mathrm{GPa}$, nitrided steel: $200 \mathrm{GPa}$ ) The displacement field holds for flat specimens, however, for specimens that are ,almost' flat the Hertzian displacement field is also expected to hold [12]. Apparent piling-up of material at the sides of the unloaded track profile completely disappeared after adding the displacement field. The shape of the lower profile resembles the shape of the indenter (dotted line). Figure 2 shows $A_{x}$ values calculated from the track width for tracks on $1 \mu \mathrm{m} \mathrm{CrN}$ coated nitrided steels (various nitriding times, various loads): $R_{\text {unl }}$ is obtained using equation (2) $A_{x}$ follows from $a$ and $R_{\text {unl }}$ using straightforward geometry. The calculated values are plotted versus measured $A_{x}$ values (numerical integration of the measured track depth profile, e.g., gray area in upper curve of figure 1). Measured $A_{x}$ values equal the calculated $A_{x}$ values. From figures 1 and 2 we conclude that unloading occurs elastically and the stress field under the sliding indenter closely resembles a Hertzian stress field.

Figure 3 shows $F_{\mathrm{f}} / F_{\mathrm{N}}$ versus $A_{x} / A_{z}$ for all specimens investigated. The data follow a straight line with slope 1 , where the data are shifted upwards by a constant value with respect to the line $F_{\mathrm{f}} / F_{\mathrm{N}}=A_{x} / A_{z}$ Consequently, equation (1) accurately describes the present experiments. The slope equals 1 , which shows that $H_{\mathrm{pl}}$ equals $H_{\mathrm{sc}}$ within experimental precision. This supports the

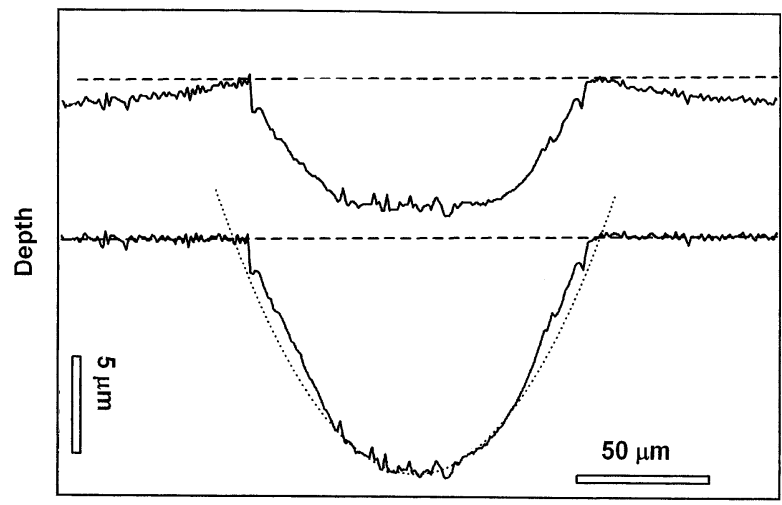

Distance across scratch track

Figure 1. Track depth profiles after unloading (upper curve) and during loading (lower curve). Gray areas denote projected contact area $A_{x}$ in the loaded and unloaded case. Dotted line: shape of the indenter. 


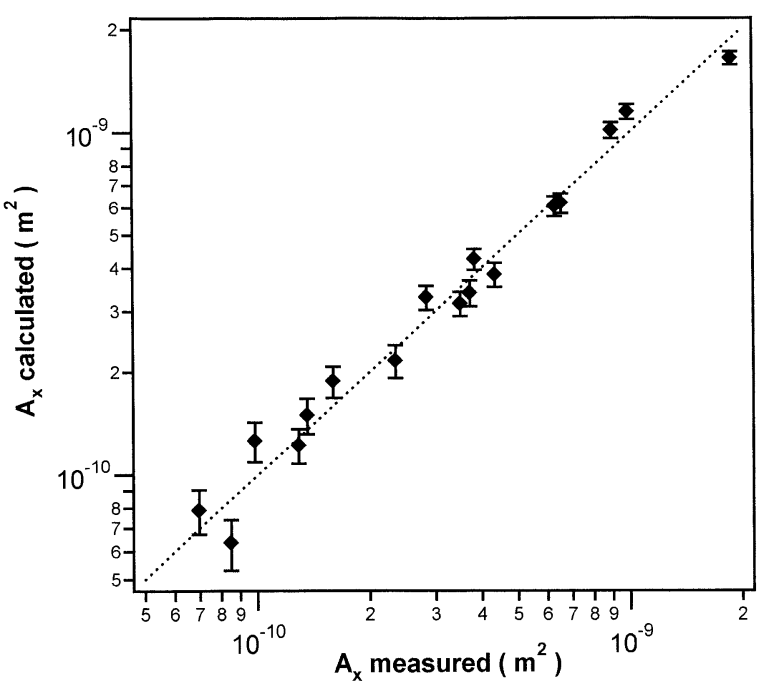

Figure 2. Calculated versus measured projected contact area $A_{x}$ dotted line: $A_{x}$ calculated $=A_{x}$ measured.

assumption that the load carrying area is approximately circular (for a load exclusively carried by the front half of the indenter a slope of approximately $1 / 2$ would be expected). In view of the absence of significant pile-up in the direct vicinity of the sides of the track during loading in figure 1 (i.e., the plastic zone is large, such that the pile-up is distributed of over relatively large area as compared to the contact area) no significant amount of material is pushed sideways during sliding: $H_{\mathrm{pl}}$ represents the resistance against deformation of material downwards. Also $H_{\mathrm{sc}}$ is associated with plastic deformation downwards, which explains that $H_{\mathrm{pl}}$ precisely equals $H_{\text {sc. }}$. The upward shift from the line $F_{\mathrm{f}} / F_{\mathrm{N}}=A_{x} / A_{z}$ in figure 3 represents $\mu_{\mathrm{sh}}$. The value $\mu_{\mathrm{sh}}$ does not depend on load, nitriding time, and the thickness of the coating: $\mu_{\text {sh }}=0.020 \pm 0.003$.

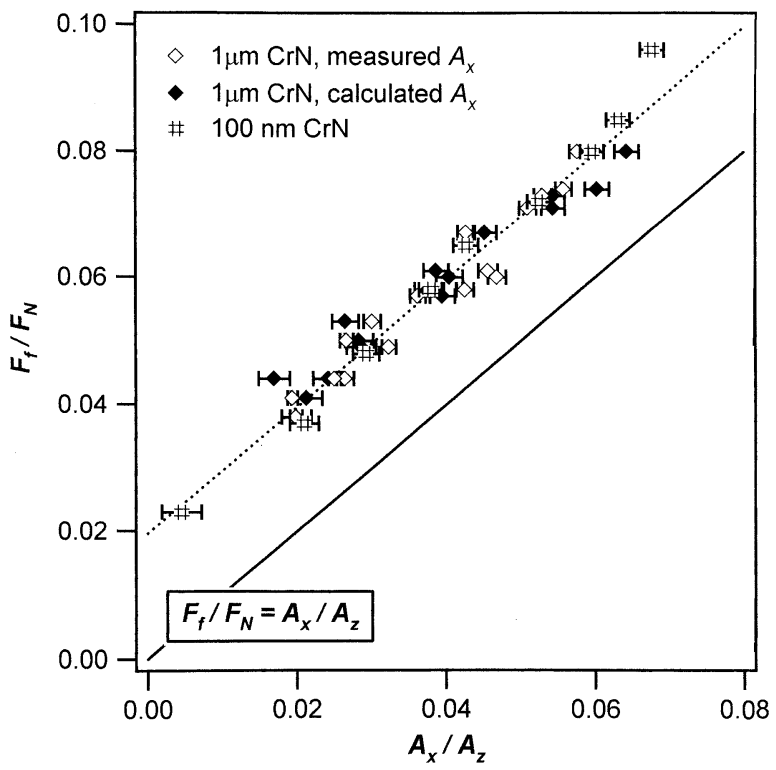

Figure 3. $F_{\mathrm{f}} / F_{x}$ versus $A_{x} / A_{z}$, solid line: $F_{\mathrm{f}} / F_{\mathrm{N}}=A_{x} / A_{z}$, dotted line: fit with slope 1 .
To further substantiate the validity of equation (1) we changed the surface condition of the $100 \mathrm{~nm} \mathrm{CrN}$ coated specimen. Changing the surface condition should not affect the plowing component, but alters the value of $\mu_{\mathrm{sh}}$. The data of the $100 \mathrm{~nm} \mathrm{CrN}$ coated specimen in figure 3 were obtained from sliding tests performed on as-deposited specimens that were in an ambient environment for various weeks before the sliding tests were performed. Additional sliding tests were performed on the same specimen that was dried shortly before the test (ultrasonic cleaning in acetone and ethanol), and for the specimen covered with a solid lubricant. For the three surface conditions, the track widths showed identical dependence on load: the plowing component was unaffected. In figure 4 the shear friction coefficient obtained after subtracting the plowing component from $F_{\mathrm{f}} / F_{\mathrm{N}}$ is plotted for the three surface conditions. Load independent shear friction is obtained. Both drying and the solid , lubricant' yield increased shear friction (in this case the lubricant does not decrease friction the friction of the diamond indenter on the ,unlubricated' $\mathrm{CrN}$ is already very low). Shear friction coefficients $\mu_{\mathrm{sh}}$ of $0.029 \pm 0.002$ and $0.034 \pm 0.002$ were found, respectively.

The present experiments were performed under relatively mild scratching conditions (track to indenter ratios of $0.1-0.4$, corresponding to contact angles of $6-24^{\circ}$ during loading). The analysis will fail, if the contact angle becomes too large (a transition from sliding to cutting can be expected) or if the elastic deformation becomes negligible with respect to the plastic deformation (projected contact area $A_{z}$ will not be circular).

In summary, we showed how elastic recovery is incorporated in the analysis of friction of a spherical indenter sliding on a flat specimen. Elastic recovery was successfully described with the expression available for a static indenter (i.e., equation (2) [12,13]). Taking into account elastic recovery affects the prevailing analyses for friction $[1,4,5]$ in two distinct ways. First, the load is not only carried by the front half of the sliding indenter,

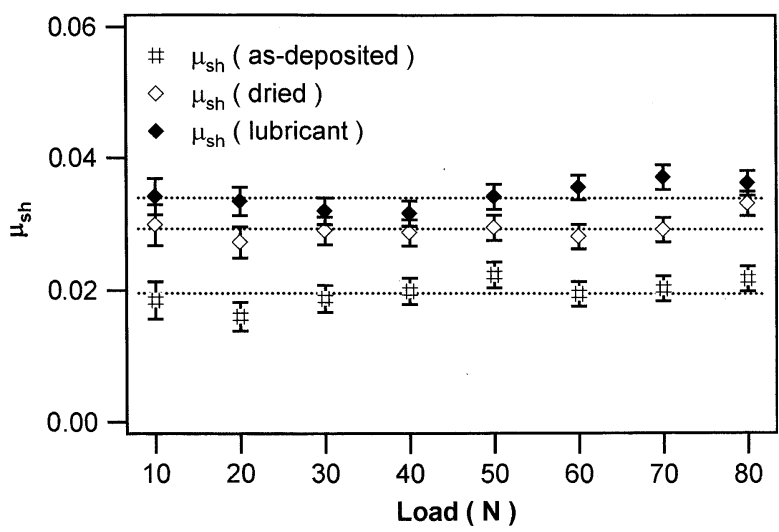

Figure 4. Shear friction coefficient $\mu_{\mathrm{sh}}$ versus load for various surface conditions 
but by both front and trailing part of the indenter. Second, instead of the geometrical contact area (projected in the sliding direction) during loading, the geometrical area after unloading determines the plowing friction force. With the proposed analysis, the shear friction coefficient $\mu_{\mathrm{sh}}$ was obtained from the track width, the indenter radius, the load, the elastic constants, and the friction force. In the present experiments the shear friction coefficient $\mu_{\mathrm{sh}}$ depends exclusively on the surface condition, i.e., not on load, nor on substrate hardness and thickness of the coating. Plowing friction is independent on surface condition. With the proposed analysis the separate contributions of substrate and coating on friction were extracted.

\section{Acknowledgment}

This research was carried out under project number MC7.01087 in the framework of the Strategic Research program of the Netherlands Institute for Metals Research in The Netherlands (www.nimr.nl).

\section{References}

[1] F.P. Bowden and D. Tabor, The Friction and Lubrication of Solids (Oxford University Press, 2001).

[2] E. Meyer, R.M. Overney, K. Dransfeld and T. Gyalog, Nanoscience: Friction and Rheology on the Nanometer Scale (World Scientific Singapore, 1998).

[3] W.P. Vellinga and C.P. Hendriks, Phys. Rev. E 63 (2001) 066121.

[4] N.P. Suh, Tribophysics (Prentice-Hall, Inc, Englewood Cliffs, New Jersey, USA, 1986).

[5] Z. Liu, J. Sun and W. Shen, Tribol. Int. 35 (2002) 511.

[6] C. Gauthier, S. Lafaye and R. Schirrer, Tribol. Int. 34 (2001) 469.

[7] J.L. Bucaille, E. Felder and G. Hochstetter, Wear 249 (2001) 422.

[8] S. Lafaye, C. Gauthier and R. Schirrer, Tribol. Int. 38 (2005) 113.

[9] J.-D. Kamminga, R. Hoy, G.C.A.M. Janssen, E. Lugscheider and M. Maes, Surf. Coat. Technol. 174-175 (2003) 671

[10] R. Hoy, J.-D. Kamminga and G.C.A.M. Janssen, Surf. Coat. Technol. 200 (2006) 3856.

[11] J.-D. Kamminga, P. van Essen, R. Hoy and G.C A.M. Janssen, Tribol. Lett. 19 (2005) 65.

[12] D. Tabor, The Hardness of Metals (Oxford University Press, 2000).

[13] G.M Hamilton, Proc. Inst. Mech. Eng. 197C (1983) 53.

[14] K.L. Johnson, Contact mechanics (Cambridge University Press, 1985).

[15] MOLYKOTE, Dow Corning Corporation, Midland Michigan, USA. 